\title{
The Cochrane Corner in the SAMJ: Summaries of Cochrane systematic reviews for evidence-informed practice
}

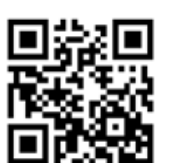

Despite substantial gains in the implementation of evidence-based practice in the past few decades, it has recently been referred to as a 'movement in crisis' ${ }^{[1]}$ This is not because the principle of using rigorous relevant evidence to inform healthcare decisions is in doubt, but rather that the ethos of evidence-based healthcare (EBHC) is being misrepresented or subverted by groups with vested interests, such as the pharmaceutical industry. The overwhelming volume of information from research and guidelines, which is difficult for practitioners to digest, as well as the notion that an evidence-based approach may undermine clinical judgement or experience, are additional barriers to the adoption of EBHC. These challenges notwithstanding, the principles of EBHC must continue to be promoted as the basis for honestly appraising the strengths and limitations of existing evidence and making informed healthcare decisions. Key to the success of EBHC is the integration of the best available, up-to-date research evidence with clinical judgement, and the incorporation of information regarding patient preferences and values. ${ }^{[2]}$

So what constitutes 'best available research evidence'? This depends on the question being asked. The randomised controlled trial remains the most reliable method for evaluating whether a treatment is effective, whereas questions about risk factors for a particular condition will best be answered by means of cohort and case-control studies. Studying patient preferences, on the other hand, will often require qualitative research. Understanding the value and limitations of each of the different research designs is therefore crucial for appropriate application of evidence in a particular context.

The systematic review is a relatively new research methodology which, along with the usual caveats regarding methodological quality, is recognised as a more trustworthy source of evidence than any single study. Unlike single studies, systematic reviews attempt to answer important healthcare questions by identifying and evaluating all relevant research studies and synthesising their results. Using rigorous and explicit methods, such reviews avoid biases resulting from 'cherry picking' certain studies and minimise the risk of being misled by studies with flawed methods, selective outcome reporting and small sample size. They also help to address publication bias through conducting comprehensive searches for both published and unpublished studies. Well-conducted, up-to-date systematic reviews therefore constitute the cornerstone of reliable evaluations of what works and what does not work in healthcare. They are now widely accepted as important for the development of evidence-informed guidelines and policies, alongside other information relevant to healthcare decision making, such as costs, feasibility and patient preferences. ${ }^{[3,4]}$

The application of systematic review methods is not limited to research addressing questions of efficacy or effectiveness, but extends to other healthcare questions including diagnosis, aetiology and prognosis, to name just a few. Methods for conducting systematic reviews of the effects of interventions, such as those produced by the Cochrane Collaboration www.cochrane.org are, however, the most developed, and typically employ rigorous approaches that have been empirically tested and validated. Furthermore, Cochrane reviews are subjected to extensive peer review, follow a standard format and are regularly updated in response to new information. ${ }^{[5,6]}$ These reviews are then published in an electronic database known as the Cochrane Database of Systematic Reviews (together with other databases of interest to healthcare decision makers) in the form of the Cochrane Library.

\section{A Cochrane Corner for the SAMJ}

The Cochrane Library currently contains more than 9000 reviews covering a wide range of healthcare topics. While these reviews are readily available for use by decision makers, busy clinicians and policy makers may not have the time to access, appraise, interpret and apply them.

This editorial introduces a regular contribution from Cochrane South Africa (http://www.mrc.ac.za/cochrane/cochrane.htm) to the South African Medical Journal, which will be called the 'Cochrane Corner. Our contribution takes the form of technical summaries of Cochrane systematic reviews handpicked for their relevance to South Africa and the African region. Our goal is to help ensure that the high-quality evidence in Cochrane reviews reaches a wider audience. We hope readers will find these future summaries helpful.

\section{Tamara Kredo}

Cochrane South Africa, South African Medical Research Council, Tygerberg, Cape Town, South Africa

Taryn Young, Charles S Wiysonge

Cochrane South Africa, South African Medical Research Council, Tygerberg, Cape Town, South Africa, and Centre for Evidence-Based Health Care, Faculty of Medicine and Health Sciences, Stellenbosch University, Tygerberg, Cape Town

\section{Michael McCaul}

Centre for Evidence-Based Health Care, Faculty of Medicine and Health Sciences, Stellenbosch University, Tygerberg, Cape Town, South Africa

\section{Jimmy Volmink \\ Cochrane South Africa, South African Medical Research Council, Tygerberg, Cape Town, South Africa, and Centre for Evidence-Based Health Care, Faculty of Medicine and Health Sciences, Stellenbosch University, Tygerberg, Cape Town}

\section{Corresponding author: T Kredo (tamara.kredo@mrc.ac.za)}

\footnotetext{
. Greenhalgh T, Howick J, Maskrey N. Evidence based medicine: A movement in crisis? BMJ 2014;348:g3725. [http://dx.doi.org/10.1136/bmj.g3725]

Sackett DL, Rosenberg WMC, Gray JAM, Haynes RB, Richardson WS. Evidence based medicine: What it is and what it isn't. BMJ 1996;312(7023):71-72. [http://dx.doi.org/10.1136/bmj.312.7023.71]

Oxman $\mathrm{AD}$, Schünemann $\mathrm{HJ}$, Fretheim $\mathrm{A}$. Improving the use of research evidence in guideline 3. Oxman AD, Schunemann H], Fretheim A. Improving the use of research evidence in guideline development: 16. Evaluation. Health Res Policy Syst 2006;4:28. [http://dx.doi.org/10.1186/1478-4505-4-28] hiv/topics/mtct/grc_handbook_mar2010_1.pdf (accessed 15 March 2015).

5. Jørgensen AW, Hilden J, Gøtzsche PC. Cochrane reviews compared with industry supported 5. Jørgensen AW, Hilden J, Gøtzsche PC. Cochrane reviews compared with industry supported
meta-analyses and other meta-analyses of the same drugs: Systematic review. BMJ 2006;333:782. http://dx.doi.org/10.1136/bmj.38973.444699.0B

6. Jadad AR, Cook DJ, Jones A, Klassen TP, Tugwell P, Moher M, Moher D. Methodology and reports of systematic reviews and meta-analyses: A comparison of Cochrane reviews with articles published in paper-based journals. JAMA 1998;280(3):278-280. [http://dx.doi.org/10.1001/jama.280.3.278]
}

S Afr Med J 2015;105(7):548. DOI:10.7196/SAMJnew.8035 